\title{
Frequency and Sociodemographic Factors Associated with Congenital Malaria at the Borgou Regional University Teaching Hospital (CHUD-B) in Benin in 2015
}

\author{
Noudamadjo Alphonse1 ${ }^{*}$, Sagbo G. Gratien², Adededmy J. Didier1, Agossou Joseph'1, \\ Obossou A. Achille1, Lokossou D. Saturnin' ${ }^{1}$ \\ ${ }^{1}$ Faculty of Medicine, University of Parakou, Parakou, Benin \\ ${ }^{2}$ Faculty of Health Sciences of Cotonou, University of Abomey-Calavi, Cotonou, Benin \\ Email: *alphonse_ndama@yahoo.fr, godsagbo@yahoo.fr, kofadier@yahoo.fr, agossoujoseph@gmail.com, \\ awadefr2000@yahoo.fr, satudolas88@gmail.com
}

How to cite this paper: Alphonse, N., Gratien, S.G., Didier, A.J., Joseph, A., Achille, O.A. and Saturnin, L.D. (2017) Frequency and Sociodemographic Factors Associated with Congenital Malaria at the Borgou Regional University Teaching Hospital (CHUD-B) in Benin in 2015. Open Journal of Pediatrics, 7, 215-221.

https://doi.org/10.4236/ojped.2017.74025

Received: September 18, 2017

Accepted: October 27, 2017

Published: October 30, 2017

Copyright (c) 2017 by authors and Scientific Research Publishing Inc. This work is licensed under the Creative Commons Attribution International License (CC BY 4.0).

http://creativecommons.org/licenses/by/4.0/

\section{(c) (i) Open Access}

\begin{abstract}
Background: Every minute a child dies from malaria in Sub-Saharan Africa. Despite this high infant and child mortality associated with malaria, congenital malaria is still not adequately understood as regarding its frequency, severity and management. The objective of this research work was to determine the frequency and identify the socio-demographic factors associated with the onset of congenital malaria at the gynecological and pediatric units of the Borgou Regional Teaching Hospital (CHUD-B) in Benin in 2015. Methods: This study was a cross-sectional survey with analytical purpose, based on exhaustive census performed from April to July 2015 on newborns delivered at CHUD-B and their mothers. The dependent variable was the presence of congenital malaria and the independent variables were socio-demographic characteristics. The data were processed and analyzed using Epi Info software version 7.0 with a significant threshold of 0.05 . Findings: The frequency of congenital malaria in newborns was $19 \%$. No sociodemographic factor was associated with congenital malaria. Conclusion: Nearly one in five newborns delivered at CHUD-B had congenital malaria. Due to malaria's contribution to perinatal morbidity and mortality and subsequent receptivity of child victims to malaria, a longer study covering a whole year and focused on the determinants of congenital malaria should be carried out.
\end{abstract}

\section{Keywords}

Congenital Malaria, Socio-Demographic Factors, Benin 


\section{Introduction}

Malaria is the most common parasitic disease globally; it mostly affects children under 5 years of age. Every two minutes, one child dies of malaria in Africa [1]. Every year, malaria causes about 2500 deaths of pregnant women and affects approximately 200,000 to 300,000 infants who suffer from consequences such as intrauterine death, low birth weight or congenital malaria [2] [3]. Despite that high infant and child mortality associated with malaria in Sub-Saharan Africa, congenital malaria is still not properly understood there as regarding its frequency, severity and management [4]. In the West African sub-region, recent studies have reported high frequencies of congenital malaria: $24.4 \%$ in Burkina-Faso [5] and $46.7 \%$ in Nigeria [6] Two studies have established a higher susceptibility to infections in general and to severe malaria in particular during the first 30 months of life in infants with history of in utero exposure to Plasmodium [7] [8]. In Benin, despite due evidence of congenital malaria's adverse effects on the infant, the national protocol for malaria management does not take into account that age group, thus giving free rein to invalidated treatments. This research work aimed to determine the frequency of congenital malaria and identify associated sociodemographic factors at CHUD-B in 2015.

\section{Methods}

This research work was a cross-sectional and descriptive study with analytical purpose carried out in the mother and child department which gathers the Gynecological \& Obstetrical and pediatric units of CHUD-B. The laboratory of the Armed Forces Teaching Hospital of Parakou served as setting for the performance of thick and thin blood smears. The study covered the period from April to July 2015. The study target population consisted of infants delivered during the study period, under 7 days of age, and their mothers. Mothers were involved while being pregnant in delivery room and after they gave their informed consent. Included in the study were all newborns born alive or not, whose mothers gave informed consent to participate. In cases of death in utero, fresh conception product of gestational age greater than 22 Weeks of Amenorrhea (SA) or weighing more than $500 \mathrm{~g}$ were also included. Excluded from the study were all newborns from HIV-infected mothers identified through systematic rapid serological screening at admission after pre-test counseling.

The sample size was determined through Schwartz formula with $\mathrm{p}=15.3 \%$ [9]. The minimal size of our study's sample was 200. Sampling was exhaustive and recruitment consecutive. The data were collected using a form completed on the basis of face-to-face interview with mothers by the delivery room staff after childbirth, and data from medical records. The variable of interest was congenital malaria. The independent variables were sociodemographic. Congenital malaria diagnosis was based on performance of thick and thin blood smears using blood from umbilical cord or peripheral blood, as well as on double-blind rapid diagnostic test (RDT). This diagnosis of congenital malaria was adopted when 
thick smear or RDT was positive. Blood thick and thin smears were performed with May Grunwald Giemsa staining technique [10] by senior lab technicians under usual working conditions in laboratory. For RDT performed by another operator in the Pediatric and Gynecological \& Obstetric units, kits of "RDT SD Bioline Malaria Ag Pf" were used.

After manual counting of data collection forms, a double entry of data was performed using Epi Data software version 3.1. The data entered were analyzed in a univariate fashion through Pearson's $\mathrm{Chi}^{2}$ test using Epi Info software version 7, with a significance threshold of 0.05 . Word processing and construction of tables have been possible using 2007 version of Microsoft Word and Excel application softwares. The measures of central tendency and dispersion (Average, Standard deviation) served to describe the quantitative variables. And for the description of the qualitative variables, ratios were used.

\section{Ethical and professional considerations}

The CHUD-B Director, the Medical Officer of the Armed Forces Teaching Hospital of Parakou and the Heads of the Gynecological \& Obstetric and Pediatric units have authorized by writing the conduct of this study. Mothers' free and informed consent was obtained before any participation. The confidentiality of data was guaranteed.

\section{Results}

\section{Characteristics of the study target population}

A total of 281 mothers and 300 infants were involved in the study. 19 mothers gave birth to twins, i.e. a twinning rate of $6.76 \%$ (19/281). There were $171(57.0 \%)$ male infants and 129 female ones (43.0\%) i.e. a sex ratio of 1.32 . The mean birth weight was $2815.4 \mathrm{~g} \pm 602.4 \mathrm{~g}$;

Mothers' mean age was $26 \pm 6.02$ years. The youngest one was 16 years and the oldest 51 years. The interval of age between 20 and 30 years was predominant. More than 3 out of 4 mothers $(76.6 \% ; \mathrm{n}=281)$ were under 30 years of age. Among the 281 mothers of infants involved, 235 lived in the city of Parakou (83.6\%) and 46 out of (16.4\%). Of the 281 mothers, 76 were housewives (27.0\%), 70 were retailers (24.9\%), 52 were artisans (18.5\%), 32 were students \%), 30 were public servants $(10.7 \%), 12$ were self-employed $(4.3 \%), 5$ were farmers $(1.8 \%)$ and four were unemployed (1.5\%).

In terms of educational attainment, 93 mothers were uneducated (33.1\%) and 188 educated (66.9\%), of whom 70 were primary (24.9\%), 96 were secondary (34.1\%) and 22 university level (7.8\%). The mothers' mean gravidity was $2.8 \pm$ 1.9 and their mean parity was $2.4 \pm 1.7$.

\section{Characteristics of infants with malaria and their mothers}

Among the 57 infants with malaria 30 were males (52.6\%) and 27 females (47.4\%) i.e. a sex ratio of 1.11 .

Their mothers' age was below 30 years in $78.9 \%$ of cases, and between 20 and 30 years in $66.7 \%$ of cases $(n=57)$. 
Table 1 shows the distribution of mothers of infants with malaria according to their place of residence, occupation and educational status.

Frequency of congenital malaria

57 out of the 300 infants involved in the study were carriers of congenital malaria, i.e. a frequency of $19.0 \%$.

Relationship between sociodemographic factors and congenital malaria

Table 2 indicates the distribution of infants according to presence in them of congenital malaria and sociodemographic factors.

\section{Discussion}

The systematic character of case recruitment may cause selection-related biases in the results of this study. Besides, the fact that the study did not cover a full year may have not made it possible to highlight seasons' influence on malaria's incidence. Eventually, the conduct of the study in a hospital setting may have introduced an effect of "concentration" of cases if interaction between malaria and pregnancy is considered. All those elements do not enable to extrapolate the results obtained on the whole hospital over a full year, even less over the whole community. However, the study has the merit of addressing a problem which is real but neglected by policy-makers in the fight against malaria.

Frequency of congenital malaria

This study has pointed out that about one out of five children born at CHUD-B in 2015 had congenital malaria. This high frequency (19.0\%) may be

Table 1. Distribution of mothers of infants with malaria delivered at CHUD-B in 2015, according to their place of residence, occupation and educational status $(n=57)$.

\begin{tabular}{ccc}
\hline & Number & Frequency (\%) \\
\hline Place of residence & 12 & 21.1 \\
Outside Parakou & 45 & 78.9 \\
In Parakou & & \\
Educational status & 19 & 33.3 \\
Uneducated & 15 & 26.3 \\
Primary school & 20 & 35.1 \\
Secondary/high school & 3 & 5.3 \\
University & & \\
Occupation & 12 & 21.1 \\
Artisan & 15 & 26.3 \\
Retailer & 5 & 8.8 \\
High school/university student & 13 & 22.8 \\
Housewife & 8 & 14.0 \\
Public servant & 4 & 7.0 \\
Liberal profession & &
\end{tabular}


Table 2. Relationship between sociodemographic factors and presence of congenital malaria in infants delivered at CHUD-B in 2015

\begin{tabular}{|c|c|c|c|c|}
\hline & \multirow{2}{*}{ Total } & \multicolumn{2}{|c|}{ Congenital malaria } & \multirow{2}{*}{$\mathrm{p}$} \\
\hline & & $\mathbf{N}$ & $\%$ & \\
\hline Sex of the infants & & & & 0.459 \\
\hline Male & 171 & 30 & 17.5 & \\
\hline Female & 129 & 27 & 20.9 & \\
\hline Place of residence & & & & 0.284 \\
\hline Outside Parakou & 49 & 12 & 24.5 & \\
\hline In Parakou & 251 & 45 & 17.9 & \\
\hline Educational status & & & & 0.861 \\
\hline Primary school & 75 & 15 & 20 & \\
\hline Secondary/high school & 103 & 20 & 19.42 & \\
\hline University & 23 & 3 & 13.04 & \\
\hline Uneducated & 99 & 19 & 19.2 & \\
\hline Mother's occupation & & & & 0.716 \\
\hline Farmer/herder & 5 & 0 & 0 & \\
\hline Artisan & 57 & 12 & 21.1 & \\
\hline Retailer & 74 & 15 & 20.3 & \\
\hline High school/university student & 34 & 5 & 14.7 & \\
\hline Housewife & 80 & 13 & 16.3 & \\
\hline Public servant & 34 & 8 & 23.5 & \\
\hline Liberal profession & 13 & 4 & 30.8 & \\
\hline Jobless & 3 & 0 & 0 & \\
\hline
\end{tabular}

due to non-use of insecticide-treated nets (ITNs) distributed free of charge to all pregnant women. It may be also due to an inadequate follow-up of intermittent preventive treatment during pregnancy (IPTp).

The frequency of congenital malaria reported by this research work is close to the one found out by Chiabi et al. (23.79\%) in 2012, during a 4-month study on congenital malaria in two infant intensive care units of Yaoundé (Cameroon). Inclusion in this study was about infants aged 0 to 7 days. Congenital malaria diagnosis was made by means of blood thick smear and additional test for infection diagnosis in order to eliminate neonatal bacterial infections [10]. By contrast, the prevalence of congenital malaria in Ile-Ife (Nigeria) reported by Obiajunwa et al. in 2005 was $46.7 \%$, twice higher than the one found out here. This frequency is the highest we have found during literature review. The study was conducted in a highly endemic area with concentration of mothers suffering from malaria, where two out of three parturient women involved had positive placental parasitemia [6].

In light of this research work and literature data, discrepancy or contrast between the epidemiological data of congenital malaria is related to lack of con- 
sensus on some elements. This variability may depend on the definition of congenital malaria, blood collection sites and time limit for blood collections in relation to childbirth, biological diagnosis methods used as well as lab technicians' experience in making and reading thick and thin blood smears' strip. It may also depend on the geographic situation of the different countries [11].

\section{Factors associated with congenital malaria}

In this same study, the sociodemographic characteristics were tested with congenital malaria without being statistically related to it. The data concerning mothers, namely place of residence $(\mathrm{p}=0.28)$, educational status $(\mathrm{p}=0.86)$ and occupation ( $\mathrm{p}=0.72$ ), had no statistical relationship with onset of congenital malaria. Moreover, no relationship between the sex of infants involved in the study and congenital malaria $(\mathrm{p}=0.459)$ was found out. We noted that most of the mothers involved in our study were young subjects $(76.6 \%)$ under 30 years of age, with a mean age of $26 \pm 6.02$ years. The ratio of women under 30 years of age becomes more significant $(79.2 \%)$ when considering mothers of infants with malaria. The same remark has been made by Nagalo et al. in Burkina Faso; they have reported that mothers were aged $27.9 \pm 5.8$ years (minima 17, maxima 41 years) on average, with a median of 27 years [5]. We did not find out in the literature sociodemographic characteristics associated with congenital malaria. Populations' behavior while coping with mosquitoes is determined at first sight by their knowledge about mechanism of malaria onset. It is therefore important to initiate studies on the relationship between the knowledge, attitude and practices of women of childbearing age concerning malaria, prevention measures and occurrence of malaria during pregnancy and by extension congenital malaria.

\section{Conclusion}

As a result of this study, it is noted that about one out of five infants born at CHUD-B carried congenital malaria. No sociodemographic factor investigated was associated with congenital malaria. A subsequent study covering a whole year is highly desirable to point out a possible seasonal variability and the level of knowledge of infant mothers in order to further enhance understanding of the factors of this disease.

\section{Acknowledgements}

We are especially grateful to the infants involved and their mothers, and to the lab technicians who have contributed to the achievement of this work.

\section{Conflict of Interest}

No conflict of interest of whatsoever nature is reported by the authors on the implementation of the research work and publication process.

\section{References}

[1] World Health Organization (2017) Malaria Aide-Memoire No. 94. https://www.who.int/mediacentre/factsheets/fs094/fr/ 
[2] World Health Organization (2008) World Malaria Report. WHO Library, Geneva, 190 p. https://www.who.int/malaria/publications/atoz/9789241563697/en/

[3] Steketee, R., Nahlen, B., Parise, M. and Menendez, C. (2001) The Burden of Malaria in Pregnancy in Malariaendemic Areas. The American Journal of Tropical Medicine and Hygiene, 64, 28-35. https://doi.org/10.4269/ajtmh.2001.64.28

[4] Uneke, C.J. (2007) Congenital Plasmodium Falciparum Malaria in Sub-Saharan Africa: A Rarity or Frequent Occurrence. Parasitology Research, 101, 835-842. https://doi.org/10.1007/s00436-007-0577-9

[5] Nagalo, K., Dao, F., Minodier, P., Sawadogo, O., Sanon, H., Tall, H.F. and Yé, D. (2014) Congenital Plasmodium falciparum Malaria: Epidemiological, Clinical, Biological, Therapeutic and Prognostic Aspects in Ouagadougou, Burkina Faso. Pan African Medical Journal, 18, 47. https://doi.org/10.11604/pamj.2014.18.47.3614 https://www.ncbi.nlm.nih.gov/pmc/articles/PMC4215357/

[6] Obiajunwa, O., Owa, J. and Adeodu, O. (2005) Prevalence of Congenital Malaria in Ile-Ife, Nigeria. Journal of Tropical Pediatrics, 51, 219-222. https://doi.org/10.1093/tropej/fmi003

[7] Rachas, A., Port, A., Cottrell, G., Guera, J., Choudat, J., Massougbodji, A., et al. (2012) Placental Malaria Associated with Increased Risk for Non-Malaria Infection during the First 18 Months of Life in a Beninese Population. Clinical Infectious Diseases, 55, 672-678. https://doi.org/10.1093/cid/cis490

[8] Schwarz, N., Adegnika, A., Breitling, L., Gabor, J., Agnandji, S., Newman, R., et al. (2008) Placental Malaria Increases Risk of Malaria during the First 30 Months of Life. Clinical Infectious Diseases, 47, 1017-1025. https://doi.org/10.1086/591968

[9] Muhktar, M.Y., Lesi, F.E.A., Iroha, E.U., Egri-Okwaji, M.T.C. and Mafe, A.G. (2006) Congenital Malaria among Inborn Babies at a Tertiary Centre in Lagos, Nigeria. Journal of Tropical Pediatrics, 52, 19-23. https://doi.org/10.1093/tropej/fmi044

[10] World Health Organization (2014) Basic Techniques for the Microscopic Diagnosis of Malaria. 2nd Edition, WHO, Geneva, $90 \mathrm{p}$. http://apps.who.int/iris/bitstream/10665/164472/1/9789242547825_fre.pdf

[11] Chiabi, A., Lendem, I., Kobela, M., Mah, E., Tietche, F. and Tchokoteu, P. (2012) Incidence of Congenital Malaria in Two Neonatal Intensive Care Units of Yaounde, Cameroon. Journal of Pediatrics and Child Care, 25, 301-308. 